OPEN ACCESS

Edited by:

Songbai Gui,

Capital Medical University, China

Reviewed by:

Liangxue Zhou,

Sichuan University, China

Todd C. Hankinson,

University of Colorado, United States

*Correspondence:

Fuyou Guo

chyou666@hotmail.com

Qi Zhang

qizhang@zzu.edu.cn

${ }^{\text {t}}$ These authors have contributed equally to this work

${ }^{\ddagger}$ These authors have contributed equally to this work

Specialty section:

This article was submitted to

Neuro-Oncology and

Neurosurgical Oncology,

a section of the journal

Frontiers in Oncology

Received: 11 July 2021

Accepted: 28 December 2021

Published: 26 January 2022

Citation:

Xu D, Guo Y, Lei S, Guo A,

Song $D$, Gao $Q$, Zhao S, Yin $K$, Wei $Q$, Zhang L, Wang $X$, Wang J,

Zhang $Q$ and Guo F (2022) Identification and Characterization of TF-IncRNA Regulatory Networks Involved in the Tumorigenesis and Development of Adamantinomatous

Craniopharyngioma.

Front. Oncol. 11:739714.

doi: 10.3389/fonc.2021.739714

\section{Identification and Characterization of} TF-IncRNA Regulatory Networks Involved in the Tumorigenesis and Development of Adamantinomatous Craniopharyngioma

\author{
Dingkang $\mathrm{Xu}^{1 \dagger}$, Yufeng Guo ${ }^{2 \dagger}$, Shixiong $\mathrm{Lei}^{1}$, Abao Guo ${ }^{1}$, Dengpan Song ${ }^{1}$, Qiang Gao ${ }^{1}$, \\ Shengqi Zhao ${ }^{1}$, Kaiwen Yin ${ }^{1}$, Qingjie Wei ${ }^{1}$, Longxiao Zhang ${ }^{1}$, Xiaoxuan Wang ${ }^{3}$, \\ Jie Wang ${ }^{3}$, Qi Zhang ${ }^{3,4^{*}}$ and Fuyou Guo ${ }^{1 * \neq}$

\begin{abstract}
${ }^{1}$ Department of Neurosurgery, The First Affiliated Hospital of Zhengzhou University, Zhengzhou, China, ${ }^{2}$ Department of Urology, First Affiliated Hospital of Zhengzhou University, Zhengzhou, China, ${ }^{3}$ Department of Pharmacology, School of Pharmaceutical Sciences, Zhengzhou University, China, Zhengzhou, China, ${ }^{4}$ State Key Laboratory of Esophageal Cancer Prevention \& Treatment, Zhengzhou University, Zhengzhou, China
\end{abstract}

Craniopharyngiomas (CPs) are rare tumors arising from the sellar region. Although the best outcome for patients with one subtype, adamantinomatous craniopharyngioma (ACP), is obtained by gross total resection, little is known about the roles of long noncoding RNAs (IncRNAs) and transcription factors (TFs) in ACP tumorigenesis. In total, 12 human ACP and 5 control samples were subjected to transcriptome-level sequencing. We built an integrated algorithm for identifying IncRNAs and TFs regulating the CP-related pathway. Furthermore, ChIP-Seq datasets with binding domain information were used to further verify and identify TF-IncRNA correlations. RT-PCR and immunohistochemistry staining were performed to validate the potential targets. Five pathways associated with ACP were identified and defined by an extensive literature search. Based on the specific pathways and the whole gene expression profile, 266 ACPrelated IncRNAs and 39 TFs were identified by our integrating algorithm. Comprehensive analysis of the ChIP-Seq datasets revealed that 29 TFs were targeted by 12000 IncRNAs in a wide range of tissues, including 161 ACP-related IncRNAs that were identified by the computational method. These 29 TFs and 161 IncRNAs, constituting 1004 TF-IncRNA pairs, were shown to potentially regulate different ACP-related pathways. A total of 232 TF-IncRNA networks were consequently established based on differential gene expression. Validation by RT-PCR and immunohistochemistry staining revealed positive expression of the ACP-related TFs E2F2 and KLF5 in ACP. Moreover, the expression of the IncRNA RP11-360P21.2 was shown to be upregulated in ACP tissues. In this study, 
we introduced an integrated algorithm for identifying IncRNAs and TFs regulating the ACP-related pathway. This is the first comprehensive study to systematically investigate the potential TF and IncRNA regulatory network in ACP. The resulting data serve as a valuable resource for understanding the mechanisms underlying ACP-related IncRNAs and TFs.

Keywords: craniopharyngiomas, LncRNA, transcription factors, RNA-seq, integrated algorithm

\section{INTRODUCTION}

Craniopharyngiomas (CPs) are benign suprasellar tumors and account for $2-4 \%$ of all intracranial tumors $(1,2)$. Despite their histological classification as WHO I tumors, CPs remain challenging to treat via total resection and postoperative management due to their specialized location and biological behavior, leading to increased mortality and poor functional results, similar to those of patients with severe endocrine disorders.

There are two types of CPs, namely, adamantinomatous craniopharyngiomas (ACPs) and papillary craniopharyngiomas (PCPs). ACPs exhibit a bimodal incidence, peaking in both childhood and at 45-60 years (1-3). The important genomic characteristics of ACPs are somatic mutations of CTNNB1, which encodes $\beta$-catenin, as revealed by widespread wholeexon sequencing, while PCPs are driven by mutation of $p$. BRAF-V600E (4). CPs harboring histological features of both ACP and PCP are extremely rare (5). Recently, multiple studies have provided new insight into the tumorigenesis of ACP and possible therapeutic targets. Mutation in exon 3 of CTNNB1, leading to overactivation of the $\mathrm{Wnt} / \beta$-catenin signaling pathway, is considered the main oncogenic driver of ACP tumorigenesis. Moreover, other pathways, such as ERBB2 and SHH signaling, have also been shown to be related to tumor growth and proliferation (6-8). Gaston et al. constructed an ACP embryonic and inducible model to further confirm that cells in the $\beta$-catenin-accumulating cluster promote tumor growth by regulating paracrine cells with a series of related proteins, including bone morphogenic proteins (BMPs) and fibroblast growth factors (FGFs) $(9,10)$. Transcriptome sequencing of murine ACP models and human tumor samples using laser capture microdissection has revealed overactivated MAPK/ERK pathways in tumor components, implying that MEK inhibitors could be developed as a potential treatment (11). Grob et al. reported high expression of IL- 6 in ACPs, and current therapies include IL- 6 inhibitors and have shown satisfactory results in patients with cystic ACP (12). The differential genetic backgrounds and epigenetic factors of ACP and PCP lead to differences in targeted therapies. Therefore, further studies at the transcription level are essential for elucidating the potential molecular mechanism of ACP.

Long noncoding RNAs (lncRNAs) are a type of RNA exceeding 200 nucleotides in length that are not translated into proteins. Mounting evidence suggests that lncRNAs impact numerous biological processes, such as cell proliferation, invasion, differentiation, apoptosis and metastasis (13).
Similarly, transcription factors (TFs) are thought to modulate the expression of lncRNAs, thereby mediating the expression of downstream molecules and promoting cancer development (14). Recently, Li et al. explored the regulatory roles of lncRNAs in different immune-related pathways in tumors using the GSEA method (15). Because ACP, which is considered a benign tumor, carries a low rate of somatic mutations, mounting evidence suggests that the TGF- $\beta$, ERBB2 and SHH signaling pathways are involved in tumor formation in an autocrine or paracrine manner. However, only a few studies focusing on the noncoding transcriptome of ACP tumors have been reported $(16,17)$.

To systematically identify the regulatory networks that are potentially associated with ACP, we utilized an integrated algorithm to specifically explore the TFs and lncRNAs regulating ACP. This is the first study to investigate TFs and lncRNAs that affect the biological behavior of ACP. In addition, we further constructed TF-lncRNA pairs, which provided new insights into the mechanism underlying ACP, and we demonstrated that the expression levels of E2F2, KLF5 and RP11-360P21.2 were significantly upregulated in ACP tissues. In conclusion, this comprehensive study on TFs and lncRNAs was performed to investigate the pathogenesis and underlying mechanism of ACP development.

\section{METHODS AND MATERIALS}

\section{Clinical Samples}

In total, 12 ACP samples and 5 normal brain tissue samples were collected from patients at the First Affiliated Hospital of Zhengzhou University, PR China. All procedures were approved by the Ethics Committee for Human Experiments of Zhengzhou University. Informed consent was obtained, and the University Review Board approved this study, which was conducted in accordance with the Helsinki Declaration. In this study, 12 ACP samples and 5 normal tissues were subjected to high-throughput RNA sequencing (RNA-seq) analysis, and 14 samples were subjected to further immunohistochemical staining. Five normal brain tissues were obtained from patients undergoing brain tissue resection due to severe traumatic intracerebral injury, and the ages and sexes of the patients in the two groups did not significantly differ.

\section{Whole RNA Sequencing}

RNA isolation and quantification were conducted according to previous reports (18). Briefly, a total of $3 \mu \mathrm{g}$ of RNA per sample was used as input material for the RNA sample preparations. 
First, ribosomal RNA was removed using an Epicenter RiboZeroTM rRNA Removal Kit (Epicenter, USA), and rRNA-free residues were removed by ethanol precipitation. Subsequently, sequencing libraries were generated using rRNA-depleted RNA with an NEBNext UltraTM Directional RNA Library Prep Kit for Illumina (NEB, USA) in accordance with the manufacturer's recommendations. The total RNA was sequenced on a HiSeq 4000 platform (Illumina, USA) at a read length of $2 \times 150 \mathrm{bp}$. Then, the limma $\mathrm{R}$ package was used to conduct differential gene expression analysis. The adjusted $\mathrm{P}$ values were analyzed to correct for false positive results in the datasets. An adjusted $\mathrm{P}<0.05$ and a $\log$ (fold change) $>1$ or log (fold change $)<-1$ were defined as the thresholds for screening differential mRNA expression.

\section{Identification of IncRNAs and TFs in ACP- Related Pathways}

Because the Wnt/ $\beta$-catenin, SHH, TGF- $\beta$, ERK1/ERK2, MAPK and ERBB2 pathways are involved in the development of $\mathrm{CP}$ $(6-8,10)$, we defined the above pathways as ACP-related pathways. To identify potential lncRNAs in ACP-related pathways, we used a calculation method involving the integration of lncRNAs and whole gene expression data. We used the method described by Li et al. with some modifications to identify TFs and lncRNAs modulating ACP (15). In brief, all genes were ranked based on their correlation with lncRNA/TF expression. To determine whether the gene sets were enriched in ACP-related pathways, the ranked genes and related pathways were calculated. The lncRNA relative enrichment score (lncRES) of each lncRNA pathway was computed, and pairs with significant values were identified. For each lncRNA or TF, we computed its activity in ACP pathways (IncRES/or TFRES) based on modified gene set enrichment analysis (GSEA).

Based on the correlation of the expression of coding genes with lncRNAs/TFs, we ranked these lncRNAs/TFs in order. Each lncRNA/or TF $\mathrm{i}$ and coding gene $\mathrm{j}$ were defined as follows: $\operatorname{lncRNA}(\mathrm{i})=(\operatorname{lncRNA} 1, \operatorname{lncRNA} 2, \operatorname{lncRNAi}, \ldots, \operatorname{lncRNAm})$ and gene $(\mathrm{j})=($ gene 1, gene 2, gene $3, \ldots$ genej,... genem $)$.

$$
\operatorname{pcorValue}(i j)=\operatorname{pearson}(i j)
$$

For each lncRNA-gene pair, the rank score (RS) was calculated as follows:

$$
R S(\mathrm{ij})=\log _{10}(p(i j)) \times p \operatorname{porValue}(i j)
$$

where $\mathrm{p}(\mathrm{ij})$ is the Pearson's (ij) $\mathrm{P}$ value.

Genes were ranked according to the RES values and subjected to enrichment analysis. To analyze the regulatory network(s) between lncRNAs and ACP-related pathways, we mapped the genes to the rank list according to the principles of GSEA. Then, the enrichment score (ES) based on the GSEA was calculated. $\mathrm{ES}_{\mathrm{ik}}$ was defined as the ES between the lncRNA $\mathrm{i}$ and ACPrelated pathway k. Finally, we integrated the $\mathrm{P}$ value and the ES into lncRES values as follows.

$$
\operatorname{LncRES}(i, k)=\left\{\begin{array}{l}
1-2 p ; \text { if } E S(i k)>0 \\
2 p-1 ; \text { if } E S(i k)<0
\end{array}\right.
$$

Consequently, the lncRESs ranged from -1 to 1 , and an absolute lncRES $>0.995$ and a false discovery rate (FDR) $<0.05$ were considered to indicate significant lncRNA-pathway pairs. The TF pathways were constructed with the same method described above. To acquire a relatively reasonable number of TFs, TFs with a RES $>0.990$ were considered significant.

\section{Identification of TF-IncRNA Regulatory Interactions}

To identify potential TF-lncRNA relationships, we downloaded a large number of ChIP-seq peak datasets of TFs from the ChIPBase database (http://rna.sysu.edu.cn/chipbase/) $(14,19)$. In ChIPBase v2.0, 10200 peak datasets generated from ChIP-seq, ChIP-exo and MNChIP-seq datasets were curated from the NCBI GEO database, ENCODE project, modENCODE project and NIH Roadmap Epigenomics project. We extracted the peak data of TFs from all lncRNA datasets and validated the peak information regarding lncRNA/TF regulators associated with ACP-related pathways. Specific TF-lncRNA pairs were established when a peak signal was observed between TFs and lncRNAs. A total of 1004 potential TF-lncRNA interactions among 29 TFs and 161 lncRNAs were generated based on the conserved TF binding sites and ChIP-Seq dataset. Consequently, we selected the significant TFs and lncRNAs from the differentially expressed genes, and a total of 232 TF-lncRNA pairs were constructed. The "OmicCircos" package (R) was used to visualize the severity of the gradients of the normalized fold changes in the expression levels of the top lncRNAs and TFs across all studies on ACP (20).

\section{Quantitative Reverse Transcription PCR Analysis (RT-PCR)}

The real-time RT-PCR method used was described in our previous study (21). Briefly, oligonucleotide primers and TaqMan probes for KLF5 and E2F2 were designed based on sequences available in the GenBank database. The PCR primer sequences designed for amplification of the target molecules are listed in the Supplemental Table. The conditions for real-time RT-PCR were as follows: preheating at $94^{\circ} \mathrm{C}$ for $2 \mathrm{~min}$, followed by 35 cycles of $94^{\circ} \mathrm{C}$ for $20 \mathrm{~s}, 60^{\circ} \mathrm{C}$ for $40 \mathrm{~s}$, and $72^{\circ} \mathrm{C}$ for $30 \mathrm{~s}$. The quantity of the $\beta$-actin gene product, a representative housekeeping gene, was equivalent in all the samples. Relative changes in gene expression were quantified using the likelihood method ( $2^{-\Delta \Delta \mathrm{Ct}}$ method). The normalized value for each target cDNA reflected the expression level of the corresponding gene.

\section{Immunohistochemistry Staining}

The immunohistochemistry method used in the present study has been previously described $(18,21)$. Briefly, sections were incubated overnight at $4^{\circ} \mathrm{C}$ with the following primary antibodies: rabbit anti-KLF5 (1:1000, Proteintech, China) and rabbit anti-E2F2 (1:1000, Proteintech, China). After washing, a goat anti-rabbit antibody (1:200, Servicebio, China) was added and incubated at room temperature. 


\section{Statistical Analysis}

Genes were considered differentially expressed if they had a fold change $\geq 2$ and a $P$ value $<0.05$. Parametric data are presented as the mean \pm standard deviation. The mean integrated optical density (IOD) was used to compare the immunohistochemical staining differences between the two groups. Differences between two groups were evaluated with the two-tailed Student's t test. All analyses were performed with $\mathrm{R}$ software (Version 3.6.3). $\mathrm{P}<0.05$ was considered statistically significant.

\section{RESULTS}

\section{Patients Characteristics and Grouping}

In total, 12 primary ACP samples and 5 control brain tissues were subjected to RNA-seq analysis. The ACP group included 5 males and 7 females, 4 of whom were children and 8 were adults $(5,7,10,13$ years old vs $22,33,51,54,56,56,57,58$ years old). The control group included 3 males and 2 females, with an average age of 40 years. There was no significant difference between the $\mathrm{ACP}$ and control groups.

\section{Identification of IncRNAs/TFs in ACP- Related Pathways}

Previous studies have indicated that the Wnt/ $\beta$-catenin, $\mathrm{SHH}$, TGF- $\beta$, Erk1/Erk2 MAPK and ERBB2 pathways play significant roles in the occurrence and development of $\mathrm{CP}$. In the present study, the identification of lncRNAs/TFs was based on ACPrelated pathways. To identify potential lncRNAs and TFs correlated with ACP-related pathways, an integrated algorithm was used to construct TF- and IncRNA-pathway networks (Figure 1A). First, the whole gene expression profiles of mRNAs and lncRNAs were obtained. Then, we calculated and ranked the RESs of genes for each lncRNA. Third, the activity of each IncRNA in the ACP-related pathway was calculated based on GSEA, and the P values were converted into lncRES values (Figure 1B). A lncRES $>0.995$ and an FDR $<0.05$ were considered significant. According to the relevant lncRNApathway pairs, the lncRNA-related pathways were in the following descending order: TGF- $\beta$, SHH, Erk1/Erk2 MAPK, ERBB2 and $W n t / \beta$-catenin (Figure 1C). In addition, the TFrelated pathways were in the following descending order: TGF- $\beta$, Wnt/ $\beta$-catenin, Erk1/Erk2 MAPK, Shh, and ERBB2 pathways (Figure 1D).

Based on the integrated algorithm, we identified TFs and lncRNAs of the ACP-related pathway, and the expression of TFs/ IncRNAs was significantly upregulated in ACP tissues (Figures 2A, B). First, 39 TFs and 266 lncRNAs were identified, and 161 of these overlapped with ChIP-Seq datasets and ACP profiles (Supplemental material, See TFRES and LncRES). Then, significant genes among the 5 signaling pathways were identified. The top 15 TF-pathway and lncRNA-pathway pairs are listed in Table 1. Finally, 1004
A

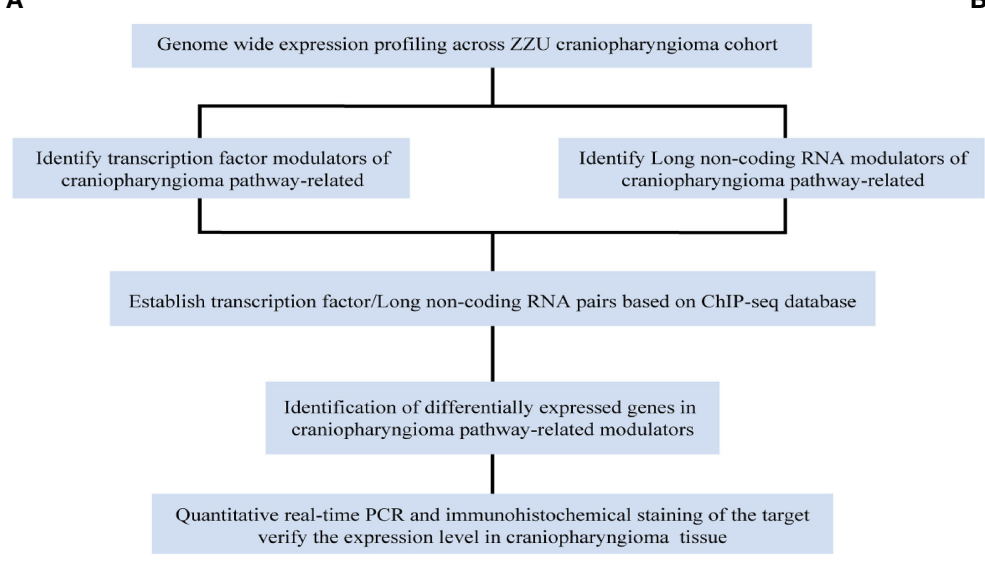

B
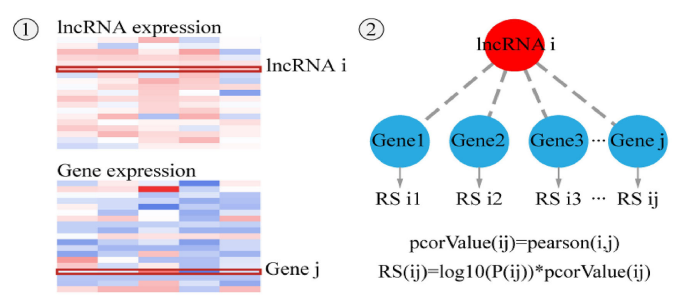

pcorValue $(\mathrm{ij})=$ pearson $(\mathrm{i}, \mathrm{j})$ $R S(\mathrm{ij})=\log 10(\mathrm{P}(\mathrm{ij})) *$ pcorValue $(\mathrm{ij})$

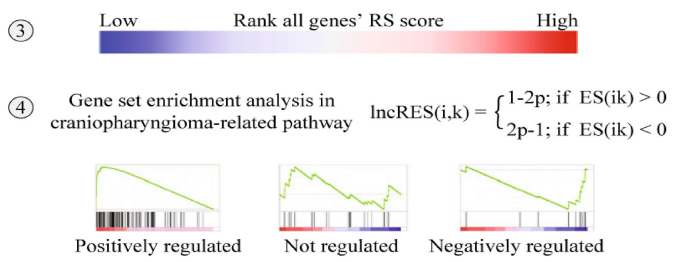

C

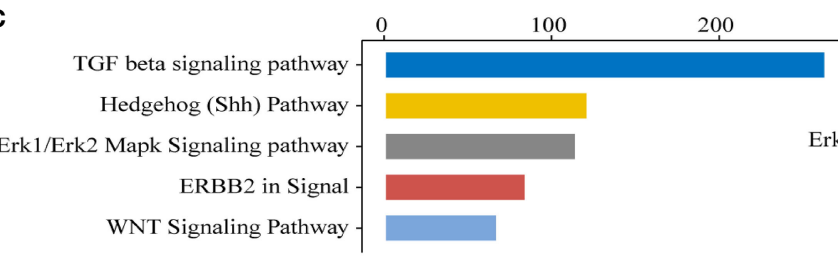

Number of Craniopharyngioma Pathway-related LncRNA

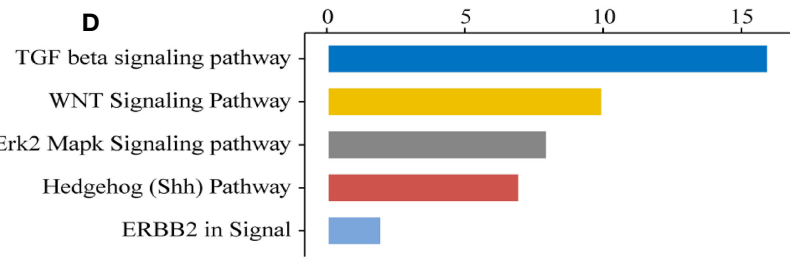

Number of Craniopharyngioma Pathway-related Transcription Factors

FIGURE 1 | Schematic of data generation and analysis (A). Workflow patterns of IncRNA-pathway and TF-pathway network construction (B). ACP pathway-related TFs and IncRNAs with significant RESs (C, D). 


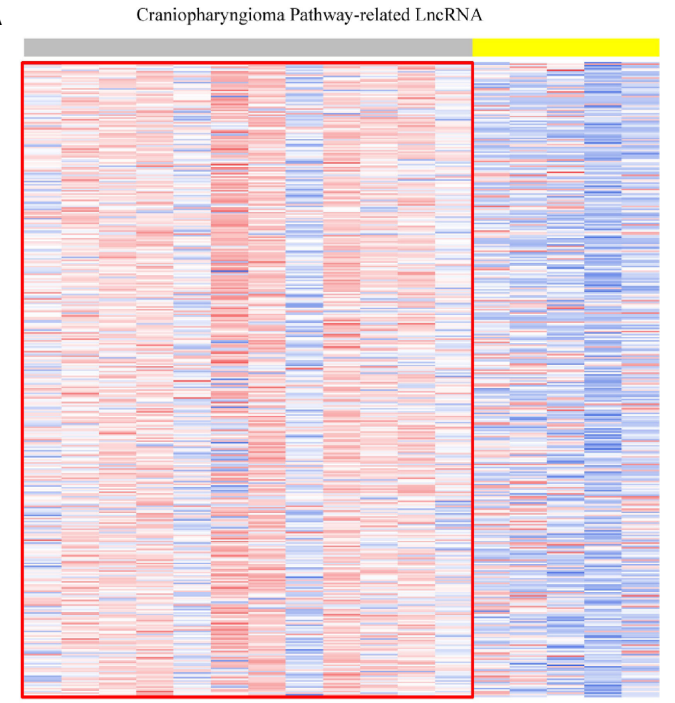

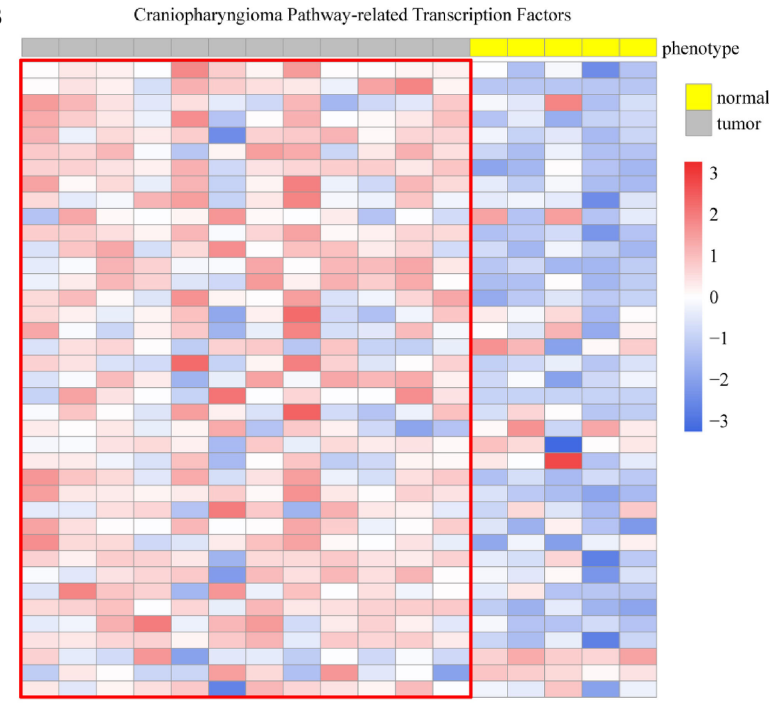

C

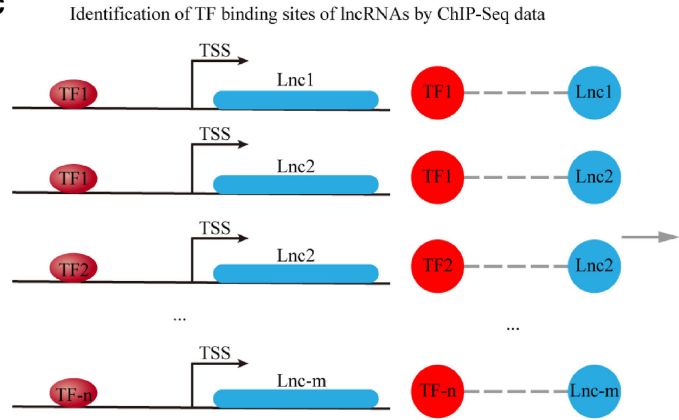

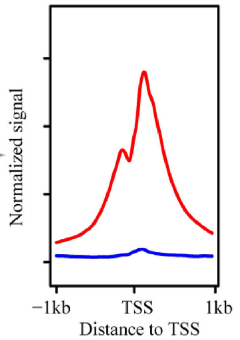

Coincidence of Craniopharyngioma Pathway-related TF and Craniopharyngioma Pathway-related LncRNA
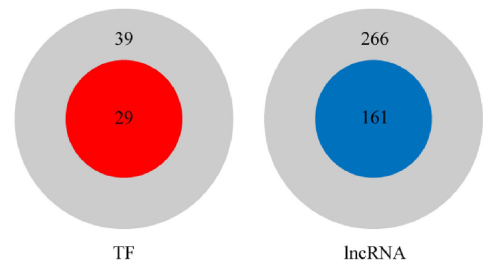

FIGURE 2 | Heatmap showing the differentially expressed ACP pathway-related IncRNAs (A) and TFs (B). The schematic diagram shows the binding process of TFs and IncRNAs (C, red represents TFs and blue represents IncRNAs). In total, 266 IncRNAs and 39 TFs were identified. Of these, 161 IncRNAs and 29 TFs with binding domain information were ultimately verified through ChIP datasets (D, red represents TFs and blue represents IncRNAs).

TF-lncRNA interactions among 29 TF pathways and 161 lncRNA pathways were constructed using the ChIP-Seq datasets (Supplemental material, see TF-lncRNA) (Figures 2C, D). In addition, the positions of the top $30 \mathrm{TFs}$ and the top 70 lncRNAs on the chromosome and their expression patterns are shown in Figure 3.

\section{Gene Expression and Construction of TF-IncRNA Pairs}

Differential gene expression analysis revealed a total of 293 RNAs that were differentially expressed. Of these RNAs, the expression levels of 59 RNAs were decreased, while the expression levels of the remaining RNAs were significantly increased (Supplemental material). The differential expression and Gene Ontology (GO) analysis were investigated between pediatric and adults (Supplemental material, see pediatric vs adults). The top 3 related biological processes (BP) were production of molecular mediator of immune response, humoral immune response and immune response-activating cell surface receptor signaling pathway (Supplemental Figure).
To explore the TF-lncRNA pairs potentially playing roles in the occurrence and development of ACP, we established a TFlncRNA network based on the ChIP-seq database. In summary, a total of 232 TF-lncRNA pairs were finally established after matching significant RNAs in the profile (Supplemental material, see TF-lncRNA pairs). For example, the STAT4 TF targeted 10 lncRNAs, and KLF5 was correlated with 25 KLF5lncRNA pairs. Of note, a pair could affect multiple pathways, and a pathway could also be affected by multiple pairs, indicating that the interaction was not one-to-one.

To further verify the reliability of the TF and lncRNA pathways we identified, we matched the data obtained from our center with those obtained from the GSE94349 dataset (Figure 4A) (22). Only the GEO cohort was used to quantify pathway activity. Although not completely significant in the ZZU dataset, which may have been due to data error and the small number of samples, all the data in the Gene Expression Omnibus (GEO) cohort were significantly correlated with the five pathways. The final TFs were exported based on the whole gene expression profile and significant differentially expressed genes (Figure 4B). 
TABLE 1 | Top 15 TFs and IncRNAs of the ACP-related pathway.

\begin{tabular}{|c|c|c|c|c|c|}
\hline & Gene & RES & FC & $\mathbf{P}$ & Pathway \\
\hline \multirow{11}{*}{ TFs } & RUNX1 & 0.99252802 & 4.835681265 & 5.29484E-07 & TGF- $\beta$ signaling pathway \\
\hline & GLI1 & 0.997775306 & 4.077460079 & 0.000782605 & Hedgehog (Shh) pathway \\
\hline & VDR & 0.992217899 & 3.747748682 & 0.00040768 & TGF- $\beta$ signaling pathway \\
\hline & DLX2 & 0.997329773 & 3.268986894 & 0.023339632 & WNT signaling pathway \\
\hline & AHR & 0.994962217 & 3.267678968 & 1.86192E-06 & TGF- $\beta$ signaling pathway \\
\hline & HOXB6 & -0.992471769 & 2.748524623 & 0.001430767 & WNT signaling pathway \\
\hline & MYC & 0.994100295 & 2.410371459 & 0.000783643 & Erk1/Erk2 Mapk signaling pathway \\
\hline & ETS1 & -0.99375 & 2.16777249 & 5.19216E-05 & Hedgehog (Shh) pathway \\
\hline & BHLHE40 & 0.997329773 & 2.012126829 & 0.001622299 & TGF- $\beta$ signaling pathway \\
\hline & ARNT2 & -1 & -1.996219981 & 0.006013635 & WNT signaling pathway \\
\hline & E2F2 & 0.992277992 & 1.861109149 & 0.00236967 & Hedgehog (Shh) pathway \\
\hline \multirow{11}{*}{ LncRNAs } & RP11-373D23.3 & 0.997641509 & 4.248671731 & 6.69968E-07 & TGF- $\beta$ signaling pathway \\
\hline & RP11-55L3.1 & -0.997903564 & 3.846392873 & 3.67095E-07 & WNT signaling pathway \\
\hline & RP4-781K5.6 & -0.997412678 & 3.673846446 & 1.65208E-06 & ERBB2 signaling pathway \\
\hline & CTB-1|21.1 & -0.995085995 & 3.474261135 & 1.29922E-06 & ERBB2 signaling pathway \\
\hline & CTC-490G23.2 & -0.997663551 & 3.437881709 & 1.65967E-06 & WNT signaling pathway \\
\hline & RP11-475I24.3 & 0.997894737 & 3.437581722 & 0.000789382 & Hedgehog (Shh) pathway \\
\hline & AC012363.13 & -0.996621622 & 3.129375139 & 9.57969E-08 & TGF- $\beta$ signaling pathway \\
\hline & RP11-452F19.3 & 0.997742664 & 3.117381748 & $5.30894 \mathrm{E}-06$ & Erk1/Erk2 Mapk signaling pathway \\
\hline & СTC-529P8.1 & -0.997572816 & -3.107091438 & 0.000117385 & ERBB2 signaling pathway \\
\hline & СТC-529P8.1 & -0.995203837 & -3.107091438 & 0.000117385 & Erk1/Erk2 Mapk signaling pathway \\
\hline & RP11-360P21.2 & -0.996632997 & 3.080472936 & $1.7834 \mathrm{E}-06$ & TGF- $\beta$ signaling pathway \\
\hline
\end{tabular}

\section{Confirmation of the Expression Changes in ACP-Related Genes}

To confirm the real expression of TFs/lncRNAs in ACPs, highly significantly expressed TFs/lncRNAs of interest were detected, including 5 TFs (KLF5, E2F2, STAT4, ETS1 and ESR1) and 7 lncRNAs. qRT-PCR was performed, revealing that the expression levels of KLF5 and E2F2 were increased in the ACP group compared with the control group $(\mathrm{p}<0.05$, Figure 5A). The expression levels of STAT4, ETS1, or ESR1 did not significantly differ between the tumor and control groups. Furthermore, the ACP samples were subjected to immunohistochemistry analysis, which demonstrated strong staining of KLF5 and E2F2 in the whole tumor tissues, especially in the cystic wall (Figure 5B). We further screened the top $5 \operatorname{lncRNA}$ pairs with the top FC values among the TFlncRNA pairs (Table 2). The expression of RP11-360P21.2 was significantly increased in ACP (Figures 5C, D). Thus, based on the PCR, immunohistochemistry, and integrated algorithm results, we established the KLF5-RP11-360P21.2 and E2F2RP11-360P21.2 pairs in ACP.

\section{DISCUSSION}

In recent years, due to the development of high-throughput technology, research on $\mathrm{CP}$ has been transferred from the genome level to the transcriptome level, but research at the nontranscriptome RNA level is still lacking in this field. Mounting evidence suggests that lncRNAs are important modulators of tumorigenesis $(23,24)$, but no research on TF/ lncRNA regulation in ACP has been performed. In this study, we established TF-lncRNA networks by using an integrated algorithm based on high-throughput sequencing data of ACP. We first summarized the ACP-related pathways and identified the pathway-related TFs and lncRNAs based on whole gene expression. Furthermore, we validated the TF-lncRNAs pairs based on exact binding domain information verified by ChIP-Seq data and found that RP11-360P21.2 was upregulated in ACP. Finally, the TF-lncRNA pairs were obtained by matching the significant differentially expressed genes. Increased KLF5 and E2F2 expression levels were observed in tumor tissues, especially in the palisading epithelium and the cyst wall. Therefore, the binding sites of KLF5-RP11-360P21.2 and E2F2-RP11-360P21.2 may become regulatory networks to mediate the expression of downstream genes playing roles in ACP development. To the best of our knowledge, this is the first study focusing on lncRNAs and TFs in ACP, and the results potentially provide pathological insight into the mechanism underlying the unique growth pattern of ACP.

The pathogenesis of $\mathrm{CP}$ is complex and involves not only driver mutations but also coding and noncoding RNAs. ACPs are a series of tumors that have low incidence rates, which limits our knowledge of these tumors at the transcriptome level, especially at the noncoding RNA level. Although our knowledge about ACP has increased, the specific regulatory 


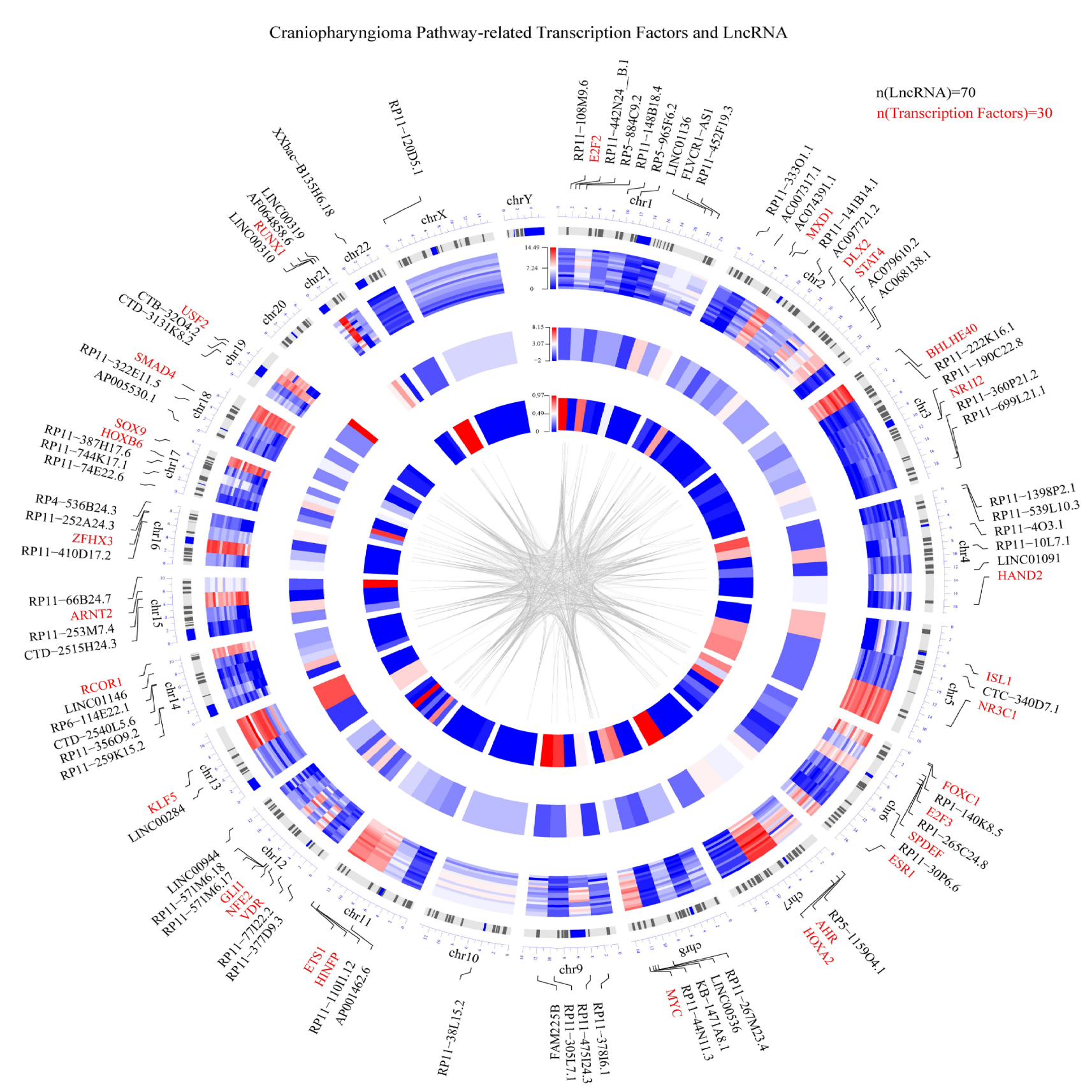

FIGURE 3 | Circular visualization of the chromosomal positions, differential expression levels and P values of the top 30 TFs and top 70 IncRNAs. The concentric circles representing different microarray datasets radiate outwards from the middle of the circle, with the $P$ values shown in the innermost group, the fold changes shown in the middle group and the differential expression of each gene shown in the outermost group. The lines from each gene symbol represent its specific chromosomal location. A weighted heatmap image was constructed according to the log2-fold changes in gene expression regardless of significance. The gray lines in the center of the circle indicate a specific interaction between the TF and IncRNA based on the ChIP datasets (red represents upregulation, and blue represents downregulation).

network of the underlying mechanism is still unclear. Increasing evidence suggests that TFs and lncRNAs play key roles in the progression of multiple cancers $(25,26)$. Therefore, the identification of TFs and lncRNAs is critical for the construction of TF-lncRNA pairs. Although several studies on CPs utilized RNA-seq analysis, most of them were limited to the expression of a few genes and lack an overall profile. Previous studies have revealed that $\mathrm{SOX} 2+$ pituitary stem cells may cause tumorigenesis in a paracrine manner based on the ACP mouse model $(9,10,23)$. Evidence indicates that the tumor as a whole is complex and that one or few molecules might not be sufficient to explore the nature of the tumor. Considering the low genomic 
A

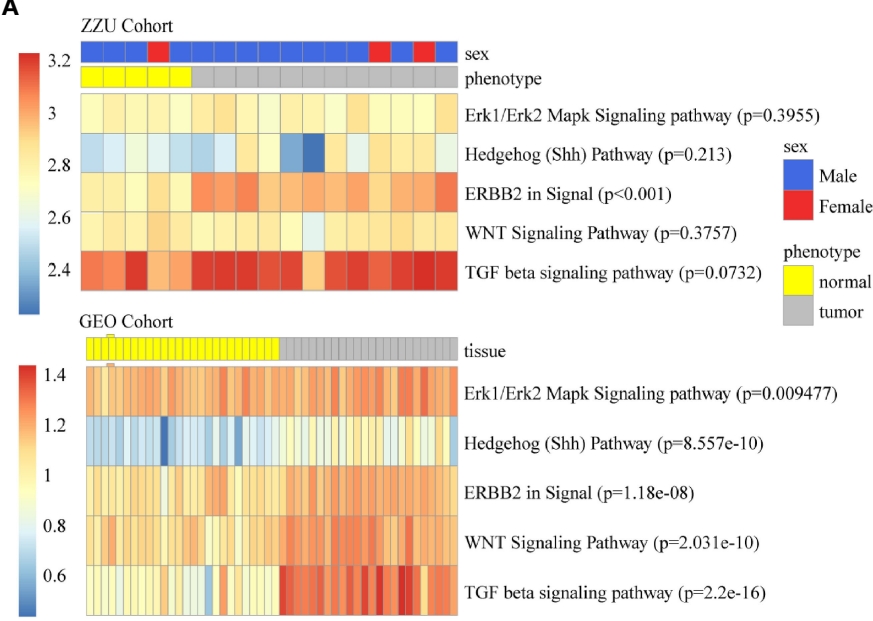

B

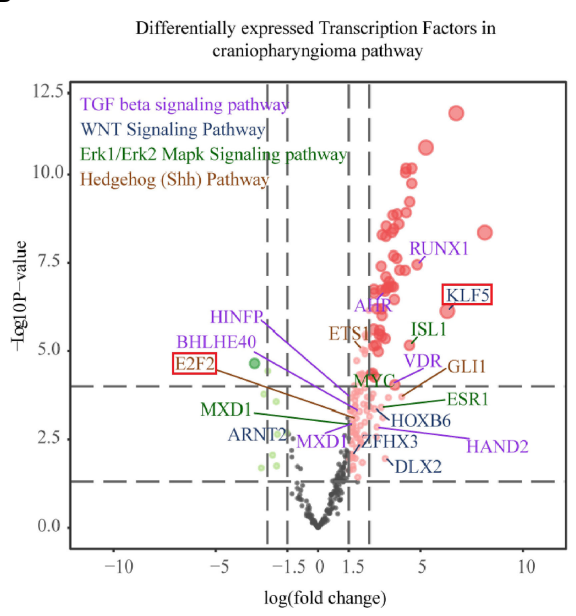

FIGURE 4 | Single-sample gene set enrichment analysis (ssGSEA) of the ZZU and GEO cohorts (A). The volcano map shows the fold changes and P values corresponding to the differentially expressed TFs among different TF-pathway pairs. The volcano plot shows the differences in mean gene expression between the $\mathrm{ACP}$ and control samples on the $\mathrm{x}$-axis and the corresponding - $\log 10$ transformed $\mathrm{p}$ values on the $\mathrm{y}$-axis. The different colors represent the various related pathways, and the colors represent the pathway with the highest RESs (B, purple represents the TGF- $\beta$ pathway, blue represents the Wnt pathway, green represents the Erk1/Erk2 pathway and brown represents the Shh pathway).

mutation rate in ACP, determining which factors among bulk gene sets that play essential roles in its biological process is difficult. Thus, we developed this method as a different validation process, and the results indicated its potential applications for prioritizing ACP-related pathway lncRNAs and TFs. The TF, lncRNA and TF-lncRNA networks have already provided novel biological insight into ACP. For instance, ESR1 yielded a high TFRES value in the ERK1/2 MAPK pathway (Table 1), indicating that it plays a preferential regulatory role in this pathway in ACP. ESR1, which is responsible for the maintenance of bone integrity, may play a key role in ACP osteogenesis. Generally, the increased activation of the ERK1/2 MAPK pathway is accompanied by the repression of ESR1 expression (27); however, the development of ACP involves the participation of both the ERK1/2 MAPK pathway and ESR1 (Table 1). This phenomenon may be attributed to the differential transcriptional backgrounds of different cell types. In addition, BMP2 was mainly expressed in the stellate reticulum and whorl-like array, while strong ERK1/2 staining was observed in the palisading epithelium. These observations suggest that these TFs and lncRNAs will be beneficial for prioritizing ACPrelated lncRNAs and TFs, leading to the identification of several novel genes as potential targets in ACP. Notably, bulk RNA sequencing might allow us to ignore some specific cellular components, and more advanced sequencing such as single-cell sequencing should serve as a new tool to better understand the tissue components in craniopharyngioma in the future.

Noncoding RNAs are emerging as critical factors involved in posttranscriptional regulation, but only a few studies have focused on this field in ACP $(16,17)$. ACP exhibits a distinct pathological structure with differential expression levels. The activation of MAPK/ERK signals occurs mainly in the palisading epithelium and reactive glial tissues, whereas BMP signaling-activated cells lie within and adjacent to $\beta$-catenin cluster cells (10). Based on previous reports, we focused more on TFs related to the processes of epidermal development, keratinocyte differentiation and odontogenesis (28). In this study, two TFs, E2F2 and KLF5, were confirmed to be differentially expressed. KLF5 encodes a member of the Krüppel-like factor zinc finger protein family and is involved in a variety of physiological processes, including proliferation, differentiation and embryogenesis. $\mathrm{Ng}$ et al. reported that KLF5 promotes tumor epithelial development in patients with Barrett's esophagus (BE) and esophageal adenocarcinoma (29). Our study revealed that KLF5 may also play the same biological role in the development of ACP. KLF5 promotes the nuclear localization and transcriptional activity of $\beta$-catenin through a physical interaction (30), revealing its crucial role in the $\mathrm{Wnt} / \beta$-catenin pathway. In addition, KLF5 binds to the RUNX2 promoter to mediate vascular smooth muscle cell calcification (31). E2F2 is a member of the E2F TF family and is mainly involved in regulating the cell cycle. Increased E2F2 expression is potentially predictive of a poor prognosis in hepatocellular carcinoma and of inflammatory cytokine upregulation $(32,33)$. In addition, several striking features were observed in the present study. Although the two TFs detected by RNA-seq are highly expressed in CPs, their expression levels differ depending on their pathological features. The expression of E2F2 and KLF5 was more prominent at the edge of the tumor cystic wall. Because the $\mathrm{CP}$ cyst is a unique feature, the cystic fluid may be a microenvironment that promotes CP growth. Previous studies have reported that inflammasomes are activated in ACP and that cholesterol crystals are potential activators, providing insight into the generation of cystic fluid and the growth pattern of ACPs $(11,23)$. The occurrence of CP may be related to several inflammatory factors, such as the IL-1- and IL-6-mediated 

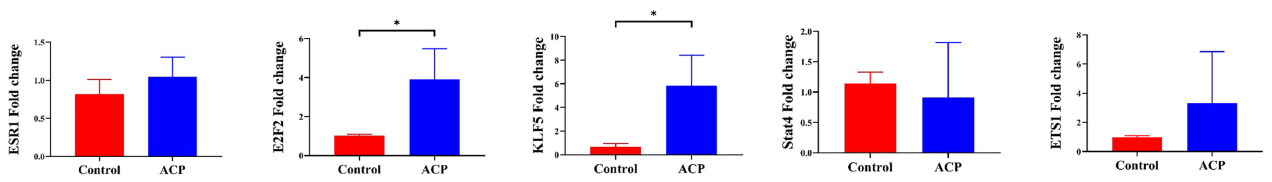

B

Normal

ACP1

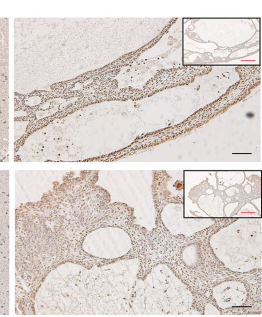

C
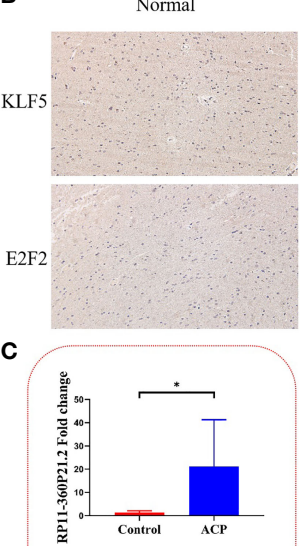

D

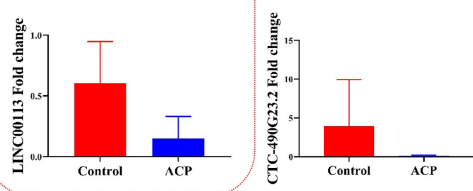

ACP2
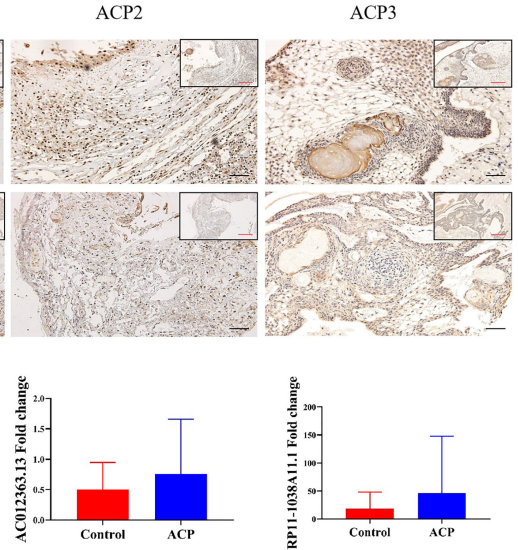
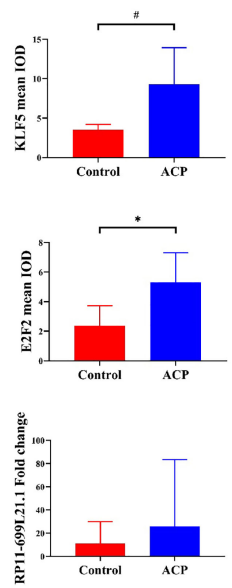

FIGURE 5 | RT-PCR analysis of ESR1, E2F2, KLF5, STAT4 and ETS1 at the transcript level. The expression of E2F2 and KLF5 was markedly increased in ACP tissues compared with normal brain tissues $(\mathbf{A}, \mathrm{n}=5$, * indicates $\mathrm{p}<0.05)$. Representative immunochemistry images of KLF5 and E2F2 in ACP. Moderate staining of KLF5 and E2F2 was observed in normal tissues (B, $n=14$, * indicates $p<0.05$, \# indicates $p<0.01)$. RT-PCR analysis revealed the expression of related IncRNAs binding to KLF5- (C) and E2F2-IncRNA pairs (D). The red box represents IncRNAs that bind to both KLF5 and E2F2. The results indicated increased expression of RP11-360P21.2 $(n=8$, * indicates $p<0.05$, \# indicates $p<0.01)$.

TABLE 2 | Top5 TF-IncRNA pairs.

\section{TF-IncRNA pairs}

Pair

KLF5-RP11-360P21.2

KLF5-RP11-699L21.1

KLF5-LINC00113

KLF5-RP11-267M23.4

KLF5-RP11-11011.12

E2F2-CTC-490G23.2

E2F2-RP11-360P21.2

E2F2-RP11-699L21.1

E2F2-LINC00113

E2F2-RP11-267M23.4

ETS1-RP11-360P21.2

ETS1-RP11-699L21.1

ETS1-RP11-267M23.4

ETS1-RP1-265C24.8

ETS1-RP11-11011.12

STAT4-RP11-699L21.1

STAT4-RP11-267M23.4

STAT4-RP1-265C24.8

ESR1-ZNF888

ESR1-RP11-35609.2

ESR1-LINC00426

ESR1-RP11-55L3.1

ESR1-CTB-1I21.1 $\log \mathrm{FC}$

adj. P. value

3.080472936

2.287542186

2.055964371

1.676662109

1.122476444

3.437881709

3.080472936

2.287542186

2.055964371

1.676662109

3.080472936

2.287542186

1.676662109

$-1.279511209$

1.122476444

2.287542186

1.676662109

$-1.279511209$

8.14568238

4.575635245

4.274391208

3.846392873

3.474261135
1.78E-06

6.55318E-05

0.000597201

0.005338561

0.016541618

1.65967E-06

1.7834E-06

$6.55318 \mathrm{E}-05$

0.000597201

0.005338561

1.7834E-06

$6.55318 \mathrm{E}-05$

0.005338561

0.021678162

0.016541618

$6.55318 \mathrm{E}-05$

0.005338561

0.021678162

8.8122E-08

8.83516E-09

5.22651E-09

3.67095E-07

1.29922E-06 
senescence-associated secretory phenotype (SASP) phenotype (23). High E2F2 expression and E2F2-RP11-360P21.2 binding may mediate downstream inflammatory factors and thus mediate the growth of CP. Due to the limitation of TF-lncRNA domain information in the CHIP-seq database, the regulatory network in CPs can be further developed using the integrated algorithm with newly acquired information in the future.

This study had several limitations, including an in-depth study on differential expressions between adults and pediatric groups, a shortage of link between pathways and phenotypes such as epithelial-mesenchymal transition $(34,35)$. In addition, the expression of more TFs and lncRNAs needs to be validated in a larger independent cohort. Future studies need to further clarify the role of the KLF5-RP11-360P21.2 and E2F2-RP11$360 \mathrm{P} 21.2$ regulatory networks in the development of ACP.

\section{CONCLUSION}

In conclusion, this study identified the potential lncRNAs and TFs in ACP and established a TF-lncRNA regulatory network in ACP at the posttranscriptional level through RNA sequencing. The present study further clarified the regulation of ACPs at the posttranscriptional level. Targeting the KLF5-RP11-360P21.2 and E2F2-RP11-360P21.2 network may serve as a novel therapeutic strategy for ACP in the future. Together, these results suggest that the identification of critical lncRNAs/TFs involved in ACP can serve as a valuable resource in the development of precision medicine.

\section{DATA AVAILABILITY STATEMENT}

The datasets generated for this study can be found in The National Omics Data Encyclopedia (NODE). Datalink: https:// www.biosino.org/node (Accession No: OER236537).

\section{ETHICS STATEMENT}

The studies involving human participants were reviewed and approved by The Ethics Committee for Human Experiments of Zhengzhou University. Written informed consent to participate

\section{REFERENCES}

1. Müller HL, Merchant TE, Warmuth-Metz M, Martinez-Barbera J-P, Puget S. Craniopharyngioma. Nat Rev Dis Primers (2019) 5:75. doi: 10.1038/s41572019-0125-9

2. Xu D, Wei Q, Li Z, Hu Y, Hu P, Zhao S, et al. Development and Validation of Predicting Nomograms for Craniopharyngioma: A Retrospective, MultipleCenter, Cohort Study. Front Oncol (2021) 11:691288. doi: 10.3389/ fonc.2021.691288

3. Guo F, Wang G, Suresh V, Xu D, Zhang X, Feng M, et al. Clinical Study on Microsurgical Treatment for Craniopharyngioma in a Single Consecutive Institutional Series of 335 Patients. Clin Neurol Neurosurg (2018) 167:162-72. doi: 10.1016/j.clineuro.2018.02.034 in this study was provided by the participants' legal guardian/ next of kin.

\section{AUTHOR CONTRIBUTIONS}

FG, DX, and YG designed the research. DX and YG performed the research and data analysis. DX, SL, QG, DS, SZ, KY, MZ, and LZ performed the basic studies. XW and JW collected the data. DX and YG wrote the paper. FG and QZ critically revised the paper. All authors contributed to the article and approved the submitted version.

\section{FUNDING}

This work was supported by grants from the National Natural Science Foundation of China (U1204807), the Science and Technology Department of Henan Province (192102310113), the Medical Science and Technique Foundation of Henan Province (SB201901007) and the Educational Department of Henan Province (19B320017).

\section{ACKNOWLEDGMENTS}

We are grateful to the patients who gracefully donated their samples to this project. We would also like to thank Dr. Zhe Li at the Second Affiliated Hospital of Zhengzhou University for collecting data during the most difficult time in the pandemic. This unforgettable memory is a spiritual treasure worth cherishing forever, and I firmly believe that mankind will eventually win the war against COVID-19.

\section{SUPPLEMENTARY MATERIAL}

The Supplementary Material for this article can be found online at: https://www.frontiersin.org/articles/10.3389/fonc.2021. 739714/full\#supplementary-material

Supplementary Figure | Enrichment analysis of differential genes between pediatric and adults. The size of the dot indicates the number of target genes, and the color represents the $p$ value.

4. Brastianos PK, Taylor-Weiner A, Manley PE, Jones RT, Dias-Santagata D, Thorner AR, et al. Exome Sequencing Identifies BRAF Mutations in Papillary Craniopharyngiomas. Nat Genet (2014) 46:161-5. doi: 10.1038/ng.2868

5. Larkin SJ, Preda V, Karavitaki N, Grossman A, Ansorge O. BRAF V600E Mutations are Characteristic for Papillary Craniopharyngioma and may Coexist With CTNNB1-Mutated Adamantinomatous Craniopharyngioma. Acta Neuropathol (2014) 127:927-9. doi: 10.1007/s00401-014-1270-6

6. Carreno G, Boult JKR, Apps J, Gonzalez-Meljem JM, Haston S, Guiho R, et al. SHH Pathway Inhibition is Protumourigenic in Adamantinomatous Craniopharyngioma. Endocr-Rel Cancer (2019) 26:355-66. doi: 10.1530/ ERC-18-0538

7. Esheba GE, Hassan AA. Comparative Immunohistochemical Expression of $\beta$ Catenin, EGFR, ErbB2, and P63 in Adamantinomatous and Papillary 
Craniopharyngiomas. J Egypt Natl Cancer Inst (2015) 27:139-45. doi: 10.1016/j.jnci.2015.06.003

8. Zuhur SS, Tanik C, Selvinaz ER, Murat MA, Fevziye K, Yuksel A. Immunohistochemical Expression of Erbb2 in Adamantinomatous Craniopharyngiomas: A Possible Target for Immunotherapy. Turk Neurosurg (2012) 23(1):55-60. doi: 10.5137/1019-5149.JTN.6706-12.1

9. Andoniadou CL, Matsushima D, Mousavy Gharavy SN, Signore M, Mackintosh AI, et al. Sox2+ Stem/Progenitor Cells in the Adult Mouse Pituitary Support Organ Homeostasis and Have Tumor-Inducing Potential. Cell Stem Cell (2013) 13:433-45. doi: 10.1016/j.stem. 2013.07.004

10. Gaston-Massuet C, Andoniadou CL, Signore M, Jayakody SA, Charolidi N, Kyeyune R, et al. Increased Wingless (Wnt) Signaling in Pituitary Progenitor/ Stem Cells Gives Rise to Pituitary Tumors in Mice and Humans. Proc Natl Acad Sci (2011) 108:11482-7. doi: 10.1073/pnas.1101553108

11. Apps JR, Carreno G, Gonzalez-Meljem JM, Haston S, Guiho R, Cooper JE, et al. Tumour Compartment Transcriptomics Demonstrates the Activation of Inflammatory and Odontogenic Programmes in Human Adamantinomatous Craniopharyngioma and Identifies the MAPK/ERK Pathway as a Novel Therapeutic Target. Acta Neuropathol (2018) 135:757-77. doi: 10.1007/ s00401-018-1830-2

12. Grob S, Mirsky DM, Donson AM, Dahl N, Foreman NK, Hoffman LM, et al. Targeting IL-6 Is a Potential Treatment for Primary Cystic Craniopharyngioma. Front Oncol (2019) 9:791. doi: 10.3389/fonc.2019.00791

13. Ransohoff JD, Wei Y, Khavari PA. The Functions and Unique Features of Long Intergenic non-Coding RNA. Nat Rev Mol Cell Biol (2018) 19:143-57. doi: $10.1038 / \mathrm{nrm} .2017 .104$

14. Guo Q, Wang J, Gao Y, Li X, Hao Y, Ning S, et al. Dynamic TF-lncRNA Regulatory Networks Revealed Prognostic Signatures in the Development of Ovarian Cancer. Front Bioeng Biotechnol (2020) 8:460. doi: 10.3389/ fbioe. 2020.00460

15. Li Y, Jiang T, Zhou W, Li J, Li X, Wang Q, et al. Pan-Cancer Characterization of Immune-Related IncRNAs Identifies Potential Oncogenic Biomarkers. Nat Commun (2020) 11:1000. doi: 10.1038/s41467-020-14802-2

16. Campanini ML, Colli LM, Paixao BM, Cabral TP, Amaral FC, Machado HR, et al. CTNNB1 Gene Mutations, Pituitary Transcription Factors, and MicroRNA Expression Involvement in the Pathogenesis of Adamantinomatous Craniopharyngiomas. Horm CANC (2010) 1:187-96. doi: 10.1007/s12672-010-0041-7

17. Samis J, Vanin EF, Sredni ST, de Bonaldo F, Costa FF, Tomita T, et al. Extensive miRNA Expression Analysis in Craniopharyngiomas. Childs Nerv Syst (2016) 32:1617-24. doi: 10.1007/s00381-016-3131-1

18. Guo F, Xu D, Lin Y, Wang G, Wang F, Gao Q, et al. Chemokine CCL2 Contributes to BBB Disruption via the P38 MAPK Signaling Pathway Following Acute Intracerebral Hemorrhage. FASEB J (2020) 34:1872-84. doi: 10.1096/fj.201902203RR

19. Karolchik D. The UCSC Genome Browser Database. Nucleic Acids Res (2003) 31:51-4. doi: 10.1093/nar/gkg129

20. Loffredo LF, Abdala-Valencia H, Anekalla KR, Cuervo-Pardo L, Gottardi CJ, Berdnikovs S. Beyond Epithelial-to-Mesenchymal Transition: Common Suppression of Differentiation Programs Underlies Epithelial Barrier Dysfunction in Mild, Moderate, and Severe Asthma. Allergy (2017) 72:1988-2004. doi: 10.1111/all.13222

21. Xu D, Gao Q, Wang F, Peng D, Wang G, Wei Q, et al. Sphingosine-1Phosphate Receptor 3 is Implicated in BBB Injury via the CCL2-CCR2 Axis Following Acute Intracerebral Hemorrhage. CNS Neurosci Ther (2021) 27 (6):674-86. doi: 10.1111/cns.13626

22. Donson AM, Apps J, Griesinger AM, Amani V, Witt DA, Anderson RCE, et al. Molecular Analyses Reveal Inflammatory Mediators in the Solid Component and Cyst Fluid of Human Adamantinomatous Craniopharyngioma. J Neuropathol Exp Neurol (2017) 76:779-88. doi: 10.1093/jnen/nlx061

23. Martinez-Barbera JP, Andoniadou CL. Biological Behaviour of Craniopharyngiomas. Neuroendocrinology (2020) 110:797-804. doi: $10.1159 / 000506904$
24. Müller HL, Merchant TE, Puget S, Martinez-Barbera J-P. New Outlook on the Diagnosis, Treatment and Follow-Up of Childhood-Onset Craniopharyngioma. Nat Rev Endocrinol (2017) 13:299-312. doi: 10.1038/ nrendo.2016.217

25. Carro MS, Lim WK, Alvarez MJ, Bollo RJ, Zhao X, Snyder EY, et al. The Transcriptional Network for Mesenchymal Transformation of Brain Tumours. Nature (2010) 463:318-25. doi: 10.1038/nature08712

26. Neph S, Stergachis AB, Reynolds A, Sandstrom R, Borenstein E, Stamatoyannopoulos JA. Circuitry and Dynamics of Human Transcription Factor Regulatory Networks. Cell (2012) 150:1274-86. doi: 10.1016/ j.cell.2012.04.040

27. Otani H, Otsuka F, Takeda M, Mukai T, Terasaka T, Miyoshi T, et al. Regulation of GNRH Production by Estrogen and Bone Morphogenetic Proteins in GT1-7 Hypothalamic Cells. J Endocrinol (2009) 203:87-97. doi: 10.1677/JOE-09-0065

28. Gump JM, Donson AM, Birks DK, Amani VM, Rao KK, Griesinger AM, et al. Identification of Targets for Rational Pharmacological Therapy in Childhood Craniopharyngioma. Acta Neuropathol Commun (2015) 3:30. doi: 10.1186/ s40478-015-0211-5

29. Ng CK, Ma K, Cheng Y, Miyashita T, Harmon JW, Meltzer SJ, et al. KrüppelLike Factor 5 Promotes Sonic Hedgehog Signaling and Neoplasia in Barrett's Esophagus and Esophageal Adenocarcinoma. Trans Oncol (2019) 12:1432-41. doi: 10.1016/j.tranon.2019.07.006

30. McConnell BB, Bialkowska AB, Nandan MO, Ghaleb AM, Gordon FJ, Yang VW, et al. Haploinsufficiency of Krüppel-Like Factor 5 Rescues the TumorInitiating Effect of the Apc ${ }^{\text {Min }}$ Mutation in the Intestine. Cancer Res (2009) 69:4125-33. doi: 10.1158/0008-5472.CAN-08-4402

31. Zhang J, Zheng B, Zhou PP, Zhang RN, He M, Yang Z, et al. Vascular Calcification is Coupled With Phenotypic Conversion of Vascular Smooth Muscle Cells Through Klf5-Mediated Transactivation of the Runx2 Promoter. Biosci Rep (2014) 34:e00148. doi: 10.1042/BSR20140103

32. Wang S, Wang L, Wu C, Sun S, Pan J. E2F2 Directly Regulates the STAT1 and PI3K/AKT/NF-Kb Pathways to Exacerbate the Inflammatory Phenotype in Rheumatoid Arthritis Synovial Fibroblasts and Mouse Embryonic Fibroblasts. Arthritis Res Ther (2018) 20:225. doi: 10.1186/s13075-018-1713-x

33. Zeng Z, Cao Z, Tang Y. Increased E2F2 Predicts Poor Prognosis in Patients With HCC Based on TCGA Data. BMC Cancer (2020) 20:1037. doi: 10.1186/ s12885-020-07529-2

34. Qi S-T, Zhou J, Pan J, Zhang C, Silky C, Yan XR. Epithelial-Mesenchymal Transition and Clinicopathological Correlation in Craniopharyngioma: EMT and Clinicopathological Correlation. Histopathology (2012) 61:711-25. doi: 10.1111/j.1365-2559.2012.04297.x

35. Chen M, Zheng S, Liu Y, Shi J, Qi S. Periostin Activates Pathways Involved in Epithelial-Mesenchymal Transition in Adamantinomatous Craniopharyngioma. J Neurol Sci (2016) 360:49-54. doi: 10.1016/ j.jns.2015.11.042

Conflict of Interest: The authors declare that the research was conducted in the absence of any commercial or financial relationships that could be construed as a potential conflict of interest.

Publisher's Note: All claims expressed in this article are solely those of the authors and do not necessarily represent those of their affiliated organizations, or those of the publisher, the editors and the reviewers. Any product that may be evaluated in this article, or claim that may be made by its manufacturer, is not guaranteed or endorsed by the publisher.

Copyright $\odot 2022$ Xu, Guo, Lei, Guo, Song, Gao, Zhao, Yin, Wei, Zhang, Wang, Wang, Zhang and Guo. This is an open-access article distributed under the terms of the Creative Commons Attribution License (CC BY). The use, distribution or reproduction in other forums is permitted, provided the original author(s) and the copyright owner(s) are credited and that the original publication in this journal is cited, in accordance with accepted academic practice. No use, distribution or reproduction is permitted which does not comply with these terms. 